\title{
Type 1 diabetes, thyroid, gastric and adrenal humoral autoantibodies are present altogether in almost one third of adult celiac patients at diagnosis, with a higher frequency than children and adolescent celiac patients
}

Claudio Tiberti, Francesca Panimolle, Raffaele Borghini, Monica Montuori, Chiara Maria Trovato, Tiziana Filardi, Andrea Lenzi \& Antonio Picarelli

To cite this article: Claudio Tiberti, Francesca Panimolle, Raffaele Borghini, Monica Montuori, Chiara Maria Trovato, Tiziana Filardi, Andrea Lenzi \& Antonio Picarelli (2020): Type 1 diabetes, thyroid, gastric and adrenal humoral autoantibodies are present altogether in almost one third of adult celiac patients at diagnosis, with a higher frequency than children and adolescent celiac patients , Scandinavian Journal of Gastroenterology

To link to this article: https://doi.org/10.1080/00365521.2020.1754898

\section{曲 Published online: 12 May 2020.}

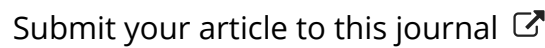

Q View related articles $\longleftarrow$

View Crossmark data ¿` 


\title{
Type 1 diabetes, thyroid, gastric and adrenal humoral autoantibodies are present altogether in almost one third of adult celiac patients at diagnosis, with a higher frequency than children and adolescent celiac patients
}

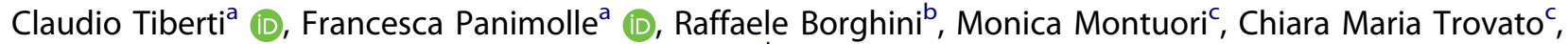 \\ Tiziana Filardi ${ }^{\mathrm{a}}$, Andrea Lenzi ${ }^{\mathrm{a}}$ and Antonio Picarelli ${ }^{\mathrm{b}}$ \\ aDepartment of Experimental Medicine, "Sapienza" University of Rome, Rome, Italy; 'Department of Internal Medicine and Clinical \\ Specialties, "Sapienza" University of Rome, Rome, Italy; 'Department of Pediatrics, Pediatric Gastroenterology and Liver Unit, "Sapienza" \\ University of Rome, Rome, Italy
}

\begin{abstract}
Background: No data are available on the frequency of organ-specific humoral autoimmunity at diagnosis of adult celiac disease (CD).

Aim: To evaluate the humoral immunoreactivities specific of type 1 diabetes (T1D), thyroid (THD), atrophic-gastritis (AG) and Addison's (AD) diseases in 92 adult CD patients at diagnosis and 237 adult healthy subjects (CTRL).

Methods: T1D, THD and AD specific autoantibodies were analyzed by radioimmunoprecipitation assays. AG autoantibodies were detected by enzyme-linked immunosorbent assay.

Results: Of $92 \mathrm{CD}$ patients, $31.5 \%$ were positive for at least one of the organ-specific autoantibodies investigated ( $p<.0001$ vs CTRL). Thyroid, diabetes, gastric and adrenal-autoantibodies, that increase with age at diagnosis, were detected in $12.0 \%, 10.9 \%, 10.9 \%, 2.2 \%$ of CD patients, respectively. Gastric- and diabetes- rather than thyroid- and adrenal-autoimmunity seem to be specifically related to presence of $C D$.

Conclusions: One third of adult CD patients at diagnosis is target of at least one organ-specific autoantibody. A systematic organ-specific autoantibody screening in these patients might be of value to promptly identify, prevent or treat the relative diseases.
\end{abstract}

ARTICLE HISTORY

Received 25 January 2020

Revised 25 March 2020

Accepted 6 April 2020

\section{KEYWORDS}

Celiac disease; organspecific autoimmunity; humoral autoimmunity

\section{Introduction}

Organ-specific autoimmune disorders are often associated with celiac disease $(\mathrm{CD})$, a chronic, systemic, gluten-dependent autoimmune disease that occurs in genetically susceptible individuals $[1,2]$. The association between $\mathrm{CD}$ and other autoimmune diseases has been widely discussed and can be explained by the sharing of a common genetic susceptibility, similar environmental triggers and the loss of intestinal barrier $[2,3]$. As it is well known [4], the presence of organ-specific autoantibodies in the serum of CD patients may be a red flag for the presence of an organ-specific disease or of an ongoing autoimmune process that can lead to the development of the disease [3,5-7]. To date, few data are available on the real frequency and co-occurrence of the organ-specific humoral autoimmune markers in $C D$ patients at diagnosis. To our best knowledge, only one study analyzed more than $20 \mathrm{CD}$ patients at diagnosis [8], whereas several others studies investigated CD patients after institution of a gluten-free diet [9-19]. The organspecific humoral autoimmune markers investigated in these studies were mainly related to type 1 diabetes [8-11] and autoimmune thyroid disease [12-16], rarely to autoimmune atrophic gastritis [17-18] and Addison's disease [11]. Recently, partially filling this void, we analyzed the humoral immunoreactivity specific to type 1 diabetes, autoimmune thyroid disease, Addison's disease and autoimmune atrophic gastritis in a large cohort of 529 biopsy-confirmed CD children and adolescents sera at diagnosis [19]. We found that $11.7 \%$ of the studied patients were positive for at least one of the antibodies investigated. To date, no data are available on the real frequency and co-occurrence of these organ-specific humoral autoimmune markers in adult CD patients at diagnosis. In this regard, this study aimed to retrospectively evaluate, in a cohort of 92 adult $C D$ patients at disease diagnosis, the humoral immunoreactivity specific of type 1 diabetes, autoimmune thyroid disease, autoimmune atrophic gastritis and Addison's disease. The results found in adult CD patients were compared to those obtained in a large cohort of adult healthy control subjects.

\section{Methods}

\section{Patients}

A total of 92 adult celiac patient sera at disease diagnosis (69 females, 23 males; age range 18-50years, median age 34.5 years) collected between 2010 and 2018 at the Department of Internal Medicine and Clinical Specialties and 
at the Department of Pediatrics, "Sapienza" University of Rome, were analyzed. At the moment of the sera collection all the $C D$ patients recruited in the study were on a glutencontaining diet. A total of $68(73.9 \%)$ CD patients presented symptoms of disease at diagnosis, including both gastrointestinal and extraintestinal manifestations, whereas 24 (26.1\%) patients were asymptomatic ( 2 subjects identified by serological screening and 22 first degree CD relatives). All the subjects enrolled in the study were screened and found positive for presence of IgA-anti-transglutaminase antibodies (IgA-tTgAb) and underwent intestinal biopsies to confirm CD diagnosis. Exclusion criteria were potential $C D$ and previous diagnosis of one or more of the four organ-specific disease investigated. Data from CD patients were compared to data from 237 adult healthy subjects (CTRL) (125 females, 122 males; age range $18.9-75.0$ years, median age 40.6 years) collected between 2010 and 2018 at Department of Experimental Medicine, "Sapienza" University of Rome. The study was approved by the Ethical Committee of "Sapienza" University of Rome.

\section{Autoantibody detection}

Celiac-specific autoimmunity: Serum IgA-tTgAb were detected by a fluid-phase radioimmunoprecipitation method reported to be the most sensitive (93\%) and specific assay (100\%) in the First International Transglutaminase Autoantibody Workshop for celiac disease [20-21].

Type 1 diabetes-specific autoimmunity: in order to simplify, speed up and considerably reduce the costs of screening for diabetes-associated humoral autoimmunity, the immune response directed against four pancreatic islet proteins specific to autoimmune diabetes [insulin (Ins), glutamic acid decarboxylase (GAD), tyrosine phosphatase $2_{(605-979)}$ (IA$\left.2 \mathrm{ic}_{(605-979)}\right)$, and islet beta-cell zinc cation efflux transporter (ZnT8)] was measured by using a single combined fluidphase radioimmunoprecipitation assay based on the detection of the four autoantibodies in an individual assay (MAA) [22]. MAA showed $92 \%$ sensitivity and $99 \%$ specificity in the Diabetes Antibody Standardization Program (DASP) [23]. Only MAA-positive sera were subsequently tested in single assays to determine individual diabetes-specific humoral immunoreactivities. As previously reported [22], the MAA method provides direct specific information on the presence of serum InsAb, whereas GAD-, IA-2 ${ }_{(605-979)^{-}}$and ZnT8-Abs were detected by well-established radioimmunological fluid-phase methods [24-26].

Thyroid-specific autoimmunity: thyroid peroxidase autoantibodies (TPOAb) were measured by using a commercially available RIA kit (Zentech, Belgium).

Gastric-specific autoimmunity: autoantibodies against the parietal cells (APCAb) specific for autoimmune chronic atrophic gastritis were measured by a commercially available ELISA kit (Axa Diagnostics, Italy).

Adrenal-specific autoimmunity: autoantibodies against the enzyme steroid 21-hydroxylase (21-OHAb) were measured by a RIA fluid-phase method using a human recombinant $21-\mathrm{OH}$ provided by Dr Alberto Falorni (University of Perugia, Perugia,
Italy). The $21-\mathrm{OHAb}$ assay achieved $94 \%$ sensitivity and $100 \%$ specificity at the first international serum exchange for the determination of 21-hydroxylase antibodies [27].

\section{Statistics}

SAS 9.3 software was used for statistical analysis. Data were expressed as frequencies or as mean \pm standard deviation or median values. Autoantibody index mean values between two groups were compared by Mann-Whitney test. Frequency differences were compared by using the chisquared test (with Yates continuity correction) or Fisher's exact test when appropriate. The Cochran-Armitage test was used to test for trend between the frequencies.

\section{Results}

\section{Autoantibody detection}

Celiac-specific autoimmunity: serum IgA-tTgAbs were positive in all the 92 adult $C D$ patients at disease diagnosis, whereas none of the 237 CTRL was positive for celiac-specific immunoreactivity.

Overall organ-specific immunoreactivity of 92 adult CD patient, 29 (31.5\%) were positive for at least one of the organ-specific antibodies investigated, significantly more frequently than healthy control patients (CTRL 21/237, 8.9\%, $p<.0001)$. The distribution of autoantibody frequencies in CD patients and CTRL subjects is reported in Figure 1. Of the 29 adult CD patients positive for at least one investigated autoantibody, $18(62.1 \%)$ did not present clinical signs of the related disease. Twenty-five out of 29 autoantibody-positive CD patients (86.2\%) were positive for only one organ-specific autoantibody, whereas the remaining four $C D$ patients were positive for two autoantibodies (in 2 cases for APCAb/ TPOAbs, in one case for APCAb/21(OH)Abs and 21(OH)Abs/ GADAbs). No statistically significant difference was found between the prevalence of type 1 diabetes, thyroid and adrenal antibodies, but the latter are less frequent than the first two. Thyroid-specific antibodies were significantly more frequent in CTRL subjects compared to the other organ-specific antibodies investigated. In spite of the organ-specific autoantibodies being more frequent in females, this difference is not significant in our cohort $(27.2 \%$ vs $17.4 \%$ males). Similarly, the overall frequency of organ-specific autoimmunity in CD symptomatic/ asymptomatic patients was not significantly different (19/68, $27.9 \%$ vs 10/24, 41.7\%). IgA-anti-transglutaminase mean autoantibody titers of the 29 organ-specific autoantibody positive $C D$ patients were not significantly different from those of the 63 autoantibody negative CD patients (Autoantibody indexes $0.582 \pm 0.355$ vs $0.541 \pm 0.388$ ).

Thyroid-specific autoimmunity: Eleven of $92 \mathrm{CD}$ patients at diagnosis $(12.0 \%)$ were positive for TPOAb, with no significant difference compared to CTRL subjects (24/237, 10.1\%) (Figure 1). TPOAbs were the organ-specific autoantibodies found most frequently in adult CD patients, significantly more present compared to adrenal antibodies $(p=.018)$, but not with respect to type 1 diabetes and parietal cell antibodies. 
Percentage of celiac patients positive for at least one organ-specific autoantibody

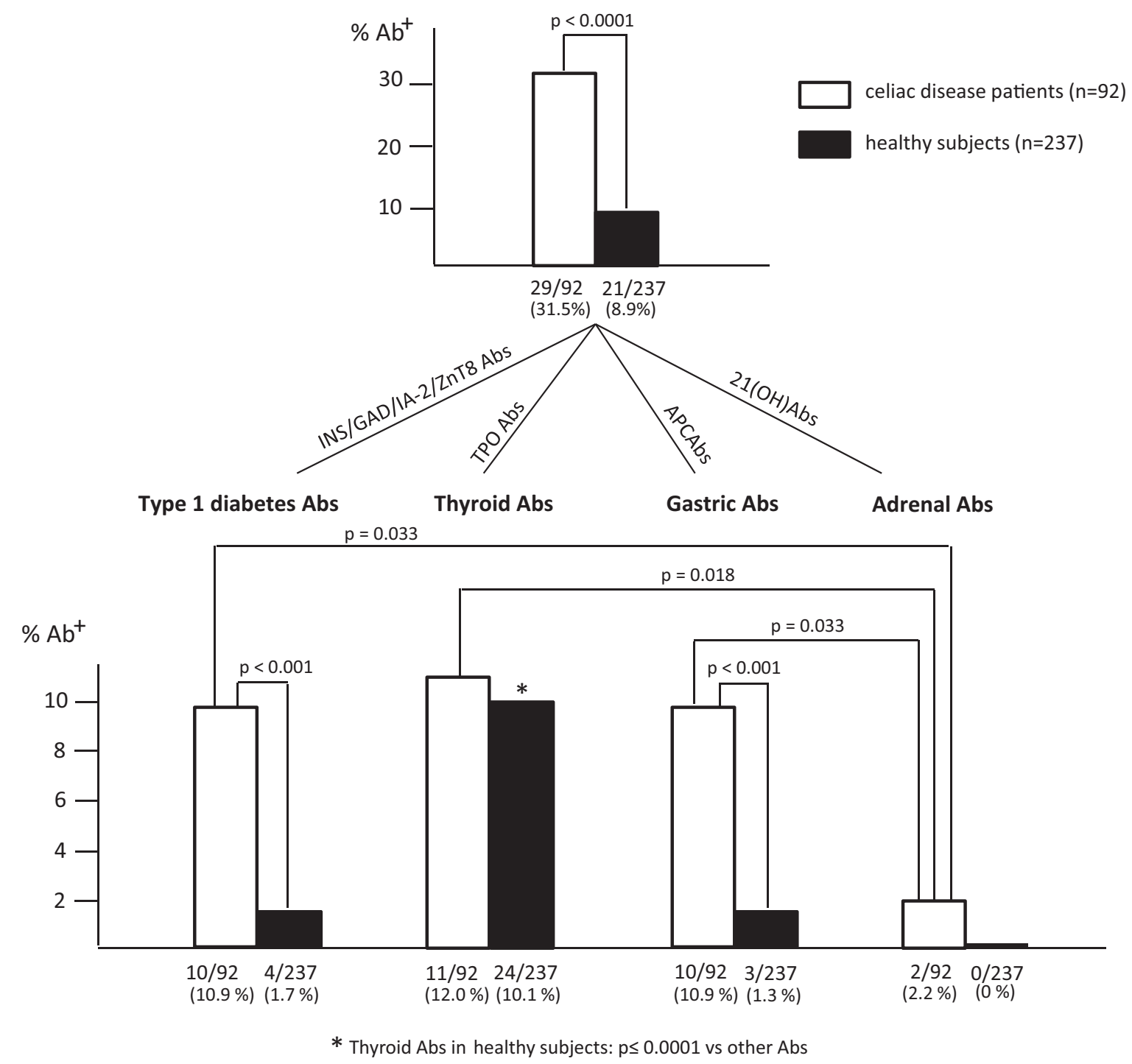

Figure 1. The upper part of the figure shows the frequency of celiac patients and healthy subjects positive for at least one of the organ-specific autoantibodies investigated in the study. The lower part of the figure shows the frequency distribution of the organ-specific autoantibody-positive celiac patients and healthy subjects according to each single disease.

Type 1 diabetes-specific autoimmunity: Ten of $92 \mathrm{CD}$ patients $(10.9 \%)$ were MAA-positive, with a significantly higher frequency relative to CTRLs $(4 / 237,1.7 \%, p=.001)$ (Figure 1). These 10 MAA-positive sera were subsequently tested in single assays to determine individual diabetes-specific humoral immunoreactivities. All the sera had autoantibodies directed against one single autoantigen. In particular, 8/10 (80.0\%) adult CD patient sera were GADAb positive, with a significantly higher frequency compared to IA-2 (1/10, $10.0 \%, p=0.005)$, ZnT8 (1/10, 10.0\%, $p=.005)$ and insulin autoantibodies $(0 / 10,0 \%, p=.001)$.

Gastric-specific autoimmunity: Of 92 adult CD patients, 10 (10.9\%) were positive for APCAb, in percentages significantly higher compared to CTRLs (3/237, 1.3\%, $p=.001)$ (Figure 1).

Adrenal-specific autoimmunity: Two of 92 adult CD patients at diagnosis (2.2\%) were positive for 21-OHAb, with no significant difference compared to CTRLs (0/237, 0\%) (Figure 1).
$C D$ organ-specific autoimmunity and age: Figure 2 shows the impact of age at diagnosis on organ-specific autoantibody frequency detected in adult $C D$ as well CTRLs. Stratifying the CD and the CTRL subjects in two groups according to median age, we found that the frequency of organ-specific autoantibodies increased, though not significantly, in CD patients with age $>34-50$ years (34.8\%) compared to younger patients (>18-34 years): 13/46 (28.3\%). Both groups of adult CD patients showed a significantly higher autoantibody frequency compared to the relative age range groups of CTRL subjects $(p<.001$ and $p=.002$, respectively).

\section{Discussion}

Recent literature reports only one study aimed to evaluate the organ-specific autoimmunity at CD diagnosis [19]. This 


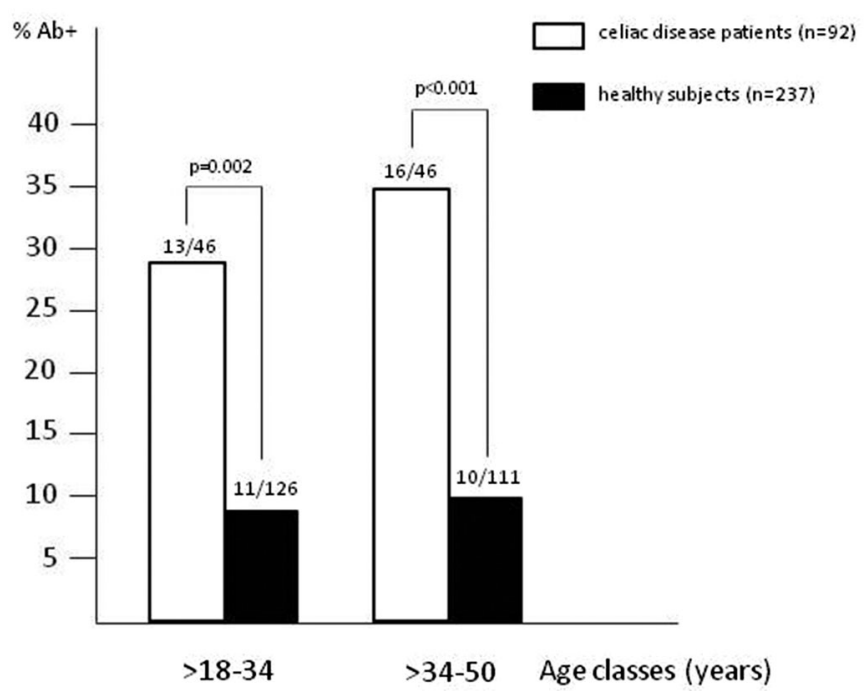

Figure 2. Impact of age at diagnosis on frequency of organ-specific autoantibodies in celiac patients and healthy subjects. The Y-axis indicate the percent of autoantibody-positive individuals (celiac patients at disease diagnosis or controls). The $x$-axis reports the CD patients and healthy subjects subdivided in four groups according to their age at diagnosis.

study was performed in our laboratory and aimed to analyze a large cohort of CD children and adolescents. With the aim of evaluating the frequency and co-occurrence of humoral organ-specific autoimmunity in adult CD patients as well, we analyzed type 1 diabetes, autoimmune atrophic gastritis, autoimmune thyroid and Addison's disease humoral immunoreactivities in 92 adult patients at $C D$ diagnosis. We found that almost one third of the adult CD patients were positive for at least one of the organ-specific autoantibodies investigated, with a significantly higher frequency compared to healthy controls. This frequency seems to be compatible with demographic and medical history data collected in a recent report [28] where $24.4 \%$ of CD patients were reported to have hypothyroidism, type 1 diabetes, autoimmune gastritis or Addison's disease. Of note, at CD diagnosis in adults we detected immunoreactivities specific of each of the four organ-specific diseases investigated. This result demonstrates the importance of screening at CD diagnosis for other autoimmune markers specific of each single disease included in the present study. We also found that only 4 (13.8\%) adult $C D$ patients were positive for more than one organ-specific disease in addition to CD. Interestingly, 3 out of 4 of these patients were positive for gastric parietal cell antibodies. A relevant percentage $(65.8 \%)$ of adult $C D$ patients at diagnosis did not present clinical signs or symptoms of the related disease; they may, however, be considered as celiac patients at risk of developing an additional organ-specific disease. Stratifying the $C D$ patients in two groups according to median age, we found that the frequency of organ-specific autoantibodies was higher in CD patients aged $>34-50$ years (34.8\%) compared with younger patients (>18-34years) (28.3\%). This increase in frequency, in itself not statistically significant, gains importance when compared to previous results from $C D$ patients aged 18 years or less (19). By combining the results of the two studies, performed by the same laboratory with the same assays, we confirmed experimentally for the first time the findings of a multicenter Italian Study [29] in which the analysis of a structured questionnaire and the review of 909 patient charts led to the conclusion that the age at diagnosis is the only significant predictor variable for the development of an additional autoimmune disease in CD patients. In particular, Ventura et al. [29] stated that patients with early diagnosed (and treated) celiac disease did not show an increased prevalence of autoimmune disorders compared to the control subjects. By contrast, CD patients diagnosed after 10 years of age showed a 7-fold higher frequency of autoimmune disorders in comparison to healthy controls. This means that the development of celiac disease at an older age is supposedly associated with a higher frequency of humoral autoimmunity specific to the organ-specific autoimmune diseases investigated. However, it is not possible to exclude that some patients with CD diagnosed later in life may have had asymptomatic disease for many years. Our data confirm a similar trend. Altogether, $31.5 \%$ of adult CD patients $>18$ years old were target of organ-specific autoimmunity, compared to $11.7 \%(p<.0001)$ of $C D$ patients $<18$ years [19]. In particular, $C D$ patients aged $>13-18$ and $>18$ years at diagnosis were target of significantly higher immunoreactivities in comparison to the same age-range group of control subjects, whereas no significant differences were found between $C D$ patients and healthy controls aged 3-8 or >8-13 years. Altogether, at disease diagnosis, thyroid, type 1 diabetes, gastric and adrenal autoantibodies were detected 2.7 times more frequently in adults than children and adolescent CD patients. However, unlike type 1 diabetes and adrenal autoantibodies, only thyroid and parietal cell autoantibody frequencies increased significantly with age. Thyroid autoantibodies are the most frequently found immune markers in adult CD patients $(12.0 \%)$, six times more than children and adolescent CD patients, but it is worth noting that they show no significant difference compared to adult healthy controls $(10.1 \%)$ that, in turn, showed a significant increase in these markers compared to children and adolescent healthy subjects (2.7\%). An increased frequency of thyroid dysfunction has already been documented in $C D$ patients, with thyroid disorders being diagnosed either before or after the diagnosis of CD [30]. Our results are in line with findings of Midhagen [31] and Counsell [32] that detected thyrotoxicosis or hypothyroidism in $10.8 \%$ and $14.0 \%$ of adult CD patients, respectively. Unlike thyroid autoimmunity, we found that adult CD patients at diagnosis show significantly higher frequencies of gastric autoantibodies $(10.9 \%)$ compared to adult healthy controls (1.3\%). Parietal cell autoantibody detection in the serum may indicate an increased risk for development of autoimmune atrophic gastritis, that can progress to pernicious anemia. Thus, presence of gastric autoantibodies may represent an important signal that will allow to evaluate, monitor in time and eventually prevent the development of chronic symptoms and irreversible complications of autoimmune atrophic gastritis in CD patients [33]. However, it is necessary to remark that these autoantibodies are not always specific for atrophic 
gastritis as they are also found in individuals with other autoimmune diseases. Adult CD patients at diagnosis also showed significantly higher frequencies of type 1 diabetes (10.9\%) autoantibodies compared to controls (1.7\%). Type 1 diabetes-specific autoimmune frequency doubles in adult $C D$ patients compared to $C D$ individuals aged less than 18 years (5.5\%). Among the type 1 diabetes autoantibodies analyzed, GAD autoantibodies, detected in $80 \%$ of patients, were significantly more present than IA-2, ZnT8 and insulin autoantibodies. Interestingly, in our study none of the adult CD patients was positive for more than one diabetes-specific autoantibody. It is well known [34] that the co-occurrence of multiple islet cell autoantibodies in a serum of a subject at risk to develop type 1 diabetes increases the risk of future development of the disease. Thus, in our study all the CD adult patients found positive for type 1 diabetes autoantibodies may be virtually considered at low-risk to develop clinical diabetes. Previous studies, based on data analysis of the Swedish national registers [35] have shown that celiac patients have a greater risk of developing Addison's disease, probably explained by the presence of common genetic traits. Despite the finding of an increased percentage of adult CD patients with adrenal autoimmunity, we found no statistically significant differences in comparison to younger patients. However, our results, with 2 organ-specific autoantibody-positive adult $C D$ patients found $21(\mathrm{OH}) \mathrm{Ab}$ positive, confirm the potential risk of Addison's disease for these patients. This finding supports the need to evaluate these autoantibodies at $\mathrm{CD}$ diagnosis, in addition to the other ones investigated in the study. In conclusion, we demonstrated that the frequencies of humoral immunoreactivities specific of type 1 diabetes, thyroid, atrophic gastritis and Addison's diseases increase with age at diagnosis of celiac disease. However, based on the comparison with healthy subjects, the increased number of adult CD patients with gastric and type 1 diabetes humoral autoimmunity appears to be more specifically related to presence of celiac disease than thyroid and adrenal autoimmunity. Amongst CD patients investigated in our study, we excluded those diagnosed for one or more of the organ-specific diseases before $C D$ diagnosis. This may give rise to the hypothesis that the coexisting autoimmunity reported in our study is likely to have been underestimated. However, our aim was to assess the frequency and co-occurrence of humoral autoimmunity specific of four organ-specific diseases in newly diagnosed adult CD patients not affected by these disorders and still not on a gluten-free diet. In light of this, the adult patients identified as target of humoral organ-specific autoimmunity at CD diagnosis may represent the basis for further studies aimed at obtaining new insights on a highly debated topic, the gluten withdrawal effects on the expression of the organ specifichumoral autoimmunity. These studies will be addressed to evaluate a) the frequency of adult patients with/without organ-specific autoimmunity at $\mathrm{CD}$ diagnosis that, despite the gluten-free diet, develop one or more of the organ-specific disease investigated b) if and possibly how the gluten-free diet changes the course and the pattern of type 1 diabetes-, thyroid-, atrophic gastritis- and Addison's humoral autoimmunity in adult CD patients. In the present study we evaluated the humoral autoimmunity related to the main organ-specific diseases that are known to be associated to CD. However, celiac disease was shown to also be associated with an autoimmune pituitary process, with ovarian failure causing infertility and with type II autoimmune polyglandular syndrome (APS, type II) although hypoparathyroidism has been rarely recorded with coincident CD [36]. It could be of interest to evaluate, at adult CD diagnosis, also humoral autoimmunity specific of these disorders as well. In conclusion, a systematic organ-specific autoantibody screening of adult CD patients might be of value to promptly identify, prevent or treat the relative diseases.

\section{Acknowledgements}

We thank Dr. Annalisa Morelli for having helped with the language revision of our work.

\section{Disclosure statement}

No potential conflict of interest was reported by the author(s).

\section{Funding}

The present study was supported by a "Sapienza" University of Rome" grant.

\section{ORCID}

Claudio Tiberti (D) http://orcid.org/0000-0002-7443-4480

Francesca Panimolle (iD) http://orcid.org/0000-0001-7639-9255

\section{References}

[1] Sollid LM, Jabri B. Triggers and drivers of autoimmunity: lessons from celiac disease. Nat Rev Immunol. 2013;13(4):294-302.

[2] Kelly CP, Bai JC, Liu E, et al. Celiac disease: clinical spectrum and management. Gastroenterology. 2015;148(6):1175-1186.

[3] Lauret E, Rodrigo L. Celiac disease and autoimmune-associated conditions. BioMed Res Int. 2013;2013:1-17.

[4] Lundin KEA, Wijmenga C. Coeliac disease and autoimmune disease-genetic overlap and screening. Nat Rev Gastroenterol Hepatol. 2015;12(9):507-515.

[5] Collin P, Kaukinen K, Välimäki M, et al. Endocrinological disorders and celiac disease. Endocrine Rev. 2002;23(4):464-483.

[6] Kahaly GJ, Schuppan D. Celiac disease and endocrine autoimmunity. Dig Dis. 2015;33(2):155-161.

[7] Diamanti A, Capriati T, Bizzarri C, et al. Celiac disease and endocrine autoimmune disorders in children. Export Rev Clin Immunol. 2013;9(12):1289-1301.

[8] Ventura A, Neri E, Ughi C, et al. Gluten-dependent diabetesrelated and thyroid-related autoantibodies in patients with celiac disease. J Pediatr. 2000;137(2):263-265.

[9] Di Mario U, Anastasi E, Mariani P, et al. Diabetes-related autoantibodies do appear in children with coeliac disease. Acta Paediatr. 1992;81(8):593-597.

[10] D'Annunzio G, Giannattasio A, Poggi E, et al. ß-cell autoimmunity in pediatric celiac disease: the case for routine screening? Diabetes Care. 2009;32(2):254-256.

[11] Toscano V, Conti FG, Anastasi E, et al. Importance of gluten in the induction of endocrine autoantibodies and organ dysfunction 
in adolescent celiac patients. Am J Gastroenterology. 2000;95(7): 1742-1748.

[12] Hadithi M, de Boer H, Meijer JWR, et al. Coeliac disease in Dutch patients with Hashimoto's thyroiditis and vice versa. WJG. 2007; 13(11):1715-1722.

[13] Ansaldi N, Palmas T, Corrias A, et al. Autoimmune thyroid disease and celiac disease in children. J Pediatr Gastroenterol Nutr. 2003; 37(1):63-66.

[14] Meloni A, Mandas C, Jores RD, et al. Prevalence of autoimmune thyroiditis in children with celiac disease and effect of gluten withdrawal. J Pediatr. 2009;155(1):51-55.

[15] Cassio A, Ricci G, Baronio F, et al. Long-term clinical significance of thyroid autoimmunity in children with celiac disease. J Pediatr. 2010;156(2):292-295.

[16] Diamanti A, Ferretti F, Guglielmi R, et al. Thyroid autoimmunity in children with coeliac disease: a prospective survey. Arch Dis Child. 2011;96(11):1038-1041.

[17] da Rosa Utiyama SR, da Silva Kotze LM, Nisihara RM, et al. Spectrum of autoantibodies in celiac patients and Relatives. Dig Dis Sci. 2001;46(12):2624-2630.

[18] Nenna R, Magliocca FM, Tiberti C, et al. Endoscopic and histological gastric lesions in children with celiac disease: mucosal involvement is not only confined to the duodenum. JPGN. 2012; 55(6):728-731.

[19] Tiberti C, Montuori M, Panimolle F, et al. Screening for type 1 diabetes-, thyroid-, gastric-, and adrenal-specific humoral autoimmunity in 529 children and adolescents with celiac disease at diagnosis identifies as positive in one of every nine patients. Dia Care. 2017;40(2):e1-e2.

[20] Bonamico M, Tiberti C, Picarelli A, et al. Radioimmunoassay to detect antitransglutaminase autoantibodies is the most sensitive and specific method for celiac disease. Am J Gastroenterology. 2001;96(5):1536-1540.

[21] $\mathrm{Li} M, \mathrm{Yu} L$, Tiberti $C$, et al. A report on the International Transglutaminase Autoantibody workshop for celiac disease. Am J Gastroenterol. 2009;104(1):154-163.

[22] Tiberti C, Yu L, Lucantoni F, et al. Detection of four diabetes-specific autoantibodies in a single radioimmunoassay: an innovative high-throughput approach for autoimmune diabetes screening. Clin Exp Immunol. 2011;166(3):317-324.

[23] Lampasona V, Schlosser M, Mueller PW, Participating Laboratories, et al. Diabetes Antibody Standardization Program: first proficiency evaluation of assays for autoantibodies to zinc transporter 8. Clin Chem. 2011;57(12):1693-1702.

[24] Grubin CE, Daniels T, Toivola B, IDDM, et al. A novel radioligand binding assay to determine diagnostic accuracy of isoform-specific glutamic acid decarboxylase antibodies in childhood. Diabetologia. 1994;37(4):344-350.

[25] Tiberti C, Verrienti A, Fiore B, et al. IA-2 combined epitope assay: a new, highly sensitive approach to evaluate IA-2 humoral autoimmunity in type 1 diabetes. Clin Immunol. 2005;115(3):260-267.

[26] Wenzlau JM, Juhl K, Yu L, et al. The cation efflux transporter $\mathrm{ZnT8}$ (SIc30A8) is a major autoantigen in human type 1 diabetes. Proc Natl Acad Sci USA. 2007;104(43):17040-17045.

[27] Falorni A, Chen S, Zanchetta R, et al. Measuring adrenal autoantibody response: Interlaboratory concordance in the first international serum exchange for the determination of 21-hydroxylase autoantibodies. Clin Immunol. 2011;140(3):291-299.

[28] Kotze LM, da Silva Kotze LR, Moreno I, et al. Immune mediated diseases in patients with celiac disease and their relatives: a comparative study of age and sex. Arq Gastroenterol. 2018;55(4): 346-351.

[29] Ventura A, Magazzù G, Greco L. Duration of exposure to gluten and risk for autoimmune disorders in patients with celiac disease. SIGEP Study Group for Autoimmune Disorders in Celiac Disease. Gastroenterology. 1999;117(2):297-303.

[30] Ch'ng CL, Keston Jones M, Kingham JGC. Celiac disease and autoimmune thyroid disease. Clin Med Res. 2007;5(3):184-192.

[31] Midhagen G, Järnerot G, Kraaz W. Adult celiac disease within a defined geographic area in Sweden. A study of prevalence and associated diseases. Scand J Gastroenterol. 1988;23(8):1000-1004.

[32] Counsell CE, Taha A, Ruddell WS. Celiac disease and autoimmune thyroid disease. Gut. 1994;35(6):844-846.

[33] Rusak E, Chobot A, Krzywicka A, et al. Anti-parietal cell antibodies - diagnostic significance. Adv.in Medical Sciences. 2016;61(2): 175-179.

[34] Ziegler AG, Rewers M, Simel O, et al. Seroconversion to multiple islet autoantibodies and risk of progression to diabetes in children. JAMA. 2013;309(23):2473-2479.

[35] Elfström P, Montgomery SM, Kämpe O, et al. Risk of primary adrenal insufficiency in patients with celiac disease. J Clin Endocrinol Metab. 2007;92(9):3595-3598.

[36] Freeman HJ. Endocrine manifestations in celiac disease. WJG. 2016;22(38):8472-8479. 